\title{
THE HYDROGEN ARRAY
}

\author{
P.N. WILKINSON \\ University of Manchester, Nuffield Radio Astronomy Laboratories, Jodrell \\ Bank, Macclesfield, Cheshire, SK11 9DL, United Kingdom
}

\begin{abstract}
The time is ripe for planning an array with a collecting area of $1 \mathrm{~km}^{2}$ (14 times larger than Arecibo and 75 times larger than the VLA). In view of its major astronomical target I have dubbed this concept 'The Hydrogen Array', although $1 \mu \mathrm{Jy}$ continuum sources will also be reliably detected. I present some initial thoughts about the issues involved.
\end{abstract}

\section{INTRODUCTION}

Since the late 1960s radioastronomers have increased the capability of their instruments many fold. The maximum resolution achieved with interferometry has increased from $\sim 50$ milliarcsec to $\sim 50$ microarcsec; the highest frequency in use has gone from $\sim 10 \mathrm{GHz}$ to $>350 \mathrm{GHz}$ and the aperture plane coverage has improved from that of the One-Mile Telescope to that of the multiconfiguration VLA. However, in terms of raw sensitivity the improvement has been less dramatic. The Arecibo telescope remains the world's largest and the improvements to system noise temperatures at decimetric and centimetric wavelengths have been relatively small $(\leq 5)$. Despite its limitations in sky and frequency coverage, the scientific output of the Arecibo telescope amply demonstrates the advantage of a collecting area 5-10 times larger than that of the largest steerable parabaloids.

Swarup and colleagues have been stressing the advantages of large collecting areas at low frequencies for over twenty years. They are now constructing the Giant Metrewave Radio Telescope (GMRT - Swarup, this volume) which is essentially a 'pointable Arecibo' working up to L-band. Pariskii (this volume) discusses the trend of collecting area with time and points out that an instrument with an area of $10^{6} \mathrm{~m}^{2}$ is now required if we are to keep up with the progress made by the pioneers. I arrived at a similar conclusion in 1985 from the following argument: the VLA and the WSRT can only image in HI with a resolution of $\sim 10 \mathrm{arcsec}$; to allow imaging at the much more useful resolution of 1 arcsec requires a telescope with $\sim 100$ times the collecting area. The reaction of colleagues at this conference to this idea convinced me that we should begin to think in earnest about a telescope with a collecting area of 1 $\mathrm{km}^{2}$. 
The first task is to establish a clear set of scientific goals. To my mind one goal stands out - a volume of the 'Encyclopaedia of the Universe' is written in $21 \mathrm{~cm}$ typescript. Unfortunately the printing is rather faint and we need a large 'lens' to read the text! Hence the reason for dubbing the proposed instrument, 'The Hydrogen Array' or HIA for short.

\section{SCIENTIFIC GOALS}

Neutral Hydrogen Observations at $z=0$ to $z=10$ : With a collecting area of $1 \mathrm{~km}^{2}$ the HIA could image nearby galaxies with a resolution of 1 arcsec. The detailed measurements of the HI distribution at this resolution would provide exciting new information on star formation, density waves and the amount and location of dark matter in individual galaxies. Some rotation curves are still flat where the surface brightness limit of current telescopes is reached. The HIA could detect galaxies $>4$ times further away than Arecibo i.e. out to $\mathrm{z}=0.1-0.2(500-1000 \mathrm{Mpc})$. Integrated $\mathrm{HI}$ observations of these galaxies will better constrain the value of $\mathrm{H}_{\mathrm{o}}$ via the Fisher-Tully technique. Deep HI redshift surveys would then measure the departures from the local Hubble flow and hence constrain the matter distribution on large scales (the 'Great Attractor' debate). Studying the kinematics of many more clusters and groups of galaxies will also constrain the total (visible+dark) matter content of the Universe. HI can be studied in absorption against background quasars out to z $>4$ with the HIA. Several quasars show Ly $\alpha$ spectra which indicate column densities $\geq 10^{20} \mathrm{~cm}^{-2}$ which should readily be detectable. Observations in the redshifted $\mathrm{HI}$ line will provide information on the structure and kinematics of the absorber which cannot be obtained in any other way. Finally a search for redshifted $\mathrm{HI}$ in emission at $\mathrm{z}=3-10$ will confront theories of galaxy formation following the era when the ionised gas combined to form HI. Observations with the HIA will be able to detect HI masses in the range $10^{12} \mathrm{M}_{\odot}$ to $10^{13} \mathrm{M}_{\odot}$.

\section{Pulsar searches and timing: The HIA would be an exciting instrument for} finding and studying pulsars, particularly millisecond ones, many of which have been found in globular clusters. The current searches with $70 \mathrm{~m}$ class telescopes have found up to 7 pulsars in a cluster, a telescope $>100$ times more sensitive would likely find an enormously larger number. Such a survey would place useful limits on the number of neutron stars in a cluster - a valuable constraint on the end-point of stellar evolution. The HIA will not only be able to find more pulsars it will also enable them to be studied in much greater detail. The accuracy of pulse timing on millisecond pulsars is limited by the achievable signal-to-noise ratio, even with Arecibo. Nevertheless timing of a few rapid pulsars in binary systems has yielded evidence for gravitational radiation and measured the absolute masses of the binary components. The periods of some isolated millisecond pulsars are extraordinarily stable. As well as producing a flood of new pulsars to study individually, an ensemble of millisecond pulsars could provide the most stable clock known to mankind. And a comparison of their periods could reveal the passage of long-wavelength gravitational radiation. Finally pulsars have already been detected in the Magellanic Clouds; with the $>100$ times greater sensitivity of the HIA it will be possible to detect pulsars in Local Group galaxies. 
Continuum Sources: If $T_{s y s}=25 \mathrm{~K}$ at $\sim 1400 \mathrm{MHz}$ and the total bandwidth is $2 \times 50=100 \mathrm{MHz}$ then the $1 \sigma$ noise level of the HIA after a four hour integration will be just over $0.1 \mu \mathrm{Jy} ; 1 \mu \mathrm{Jy}$ sources will therefore be reliably detected. With this sensitivity many observing programmes suggest themselves. For example: i) The extension of the radio luminosity function to very faint sources; do new populations of source exist? ii) How many normal galaxies have compact sources in their nuclei? VLBI between HIA and a $70 \mathrm{~m}$ class telescope would pin-point those galaxies with milliarcsec nuclear structures; the results would constrain the frequency of occurence of black holes in galactic nuclei. iii) Extragalactic supernovae could be detected $\sim 10$ times further away than is currently possible in the radio. VLBI observations with the HIA would enable radio 'hypernovae', such as $41.9+58$ in M82 and SN1986j in NGC891, to be studied out to > $500 \mathrm{Mpc}$ iv) A large number of stellar systems would be detectable with the HIA; almost certainly new types of stellar radio source would be found. Note, for example, that the quiescent flux density of the Sun at L-band would be $\sim 1 \mu \mathrm{Jy}$ at a distance of $5 \mathrm{pc}$; the flaring Sun could be detected ten times further away.

Other Targets: HIA could extend the study of $\mathrm{OH}$ mega-masing, in those galaxies currently undergoing the greatest amount of star formation, out to $\mathrm{z}=0.2-0.3$. HIA would have about one tenth the area eventually envisaged for project Cyclops. It would have obvious interest for the SETI community.

\section{TECHNICAL CONSIDERATIONS}

Array designs need to trade off cost, collecting area, primary field-of-view, complexity of electronics and imaging speed. In view of the frequency flexibility required for the scientific programme outlined above, dish antennas are preferred despite their relatively high unit cost compared with cylindrical antennas. The GMRT designers opted for parabaloids for this reason, and because of the additional data processing problems associated with cylindical elements (e.g. Gray, this volume).

Antenna Specification With an antenna diameter of $50 \mathrm{~m}$ (similar to GMRT) over 500 antennas would be required for a collecting area of $1 \mathrm{~km}^{2}$. With a diameter of $113 \mathrm{~m}$ only 100 antennas are needed; this would reduce the amount and complexity of the electronics. In order to keep the overall cost to within reasonable bounds (which I arbitrarily set at around $\$ 300 \mathrm{M}-$ comparable with the cost of the ESO VLT optical array) the unit cost of a $113 \mathrm{~m}$ antenna must therefore be around $\$ 2-2.5 \mathrm{M}$. The unit cost of the fullysteerable $45 \mathrm{~m}$ antennas of the GMRT is $\$ 0.5 \mathrm{M}$; applying a (diameter) ${ }^{2.7}$ law leads to an estimated cost of $\$ 6 \mathrm{M}$ for a $113 \mathrm{~m}$ antenna, even with the low labour costs in India. Some reduction in capability over the $45 \mathrm{~m}$ design may have to be accepted, even allowing for the greater economy of scale inherent in the HIA. The obvious parameter to relax is sky coverage but a range of $\pm 30^{\circ}$ in zenith angle is the minimum acceptable. On the other hand the design must provide full aperture efficiency at L-band, whereas the GMRT design has reduced efficiency above $\sim 1 \mathrm{GHz}$. Innovative thinking is clearly needed here. A start might be made with the SMART concept pioneered by Swarup and 
colleagues, with the parabaloid mounted on (or in) a rotatable frame, low to the ground to minimise wind loads.

Array Configuration The HIA will operate at long wavelengths (for highly redshifted HI), where the rfi environment is likely to be hostile; it should therefore be capable of producing images quickly. Circular arrays, pioneered by Wild and studied in detail by Cornwell for the $\mathrm{mm}$-wave array, seem attractive for this reason. The antenna locations must be fixed to minimise cost and the overall dimension of the array must be $\sim 40 \mathrm{~km}$ to give 1 arcsec resolution at $1420 \mathrm{MHz}$. A few more distant outriggers would extend the capability of the array for continuum work; for the latter work the array should be designed to make maximum use of multi-frequency synthesis (Conway - this volume). For pulsar observations, a central compact array containing a significant fraction of the collecting area would be needed. With an overall dimension of $1 \mathrm{~km}$ the compact array would have $\sim 100$ synthesised beam areas within the primary beam. For pulse searching each beam would need to be treated as an output from a separate telescope. The estimated data rate is not excessive for the computers of the 1990s.

Location: One of the prime considerations when deciding where to build the HIA will be the rfi environment. There is much science to be accomplished outside the protected bands, most obviously for observing neutral hydrogen at $z>0.015$. This seems to rule out Europe and the USA; snow militates against remote Canadian sites. For pulsar work one needs a good view of the galactic plane. A site in India would have the advantage of low labour costs for construction, but the rfi environment will be degraded as Indian industrialisation proceeds. The Australian outback sounds promising (pulsar searches at Parkes routinely use a bandwidth of $\sim 300 \mathrm{MHz}$ at L-band) but then there are not as many telescopes with which the HIA could do VLBI.

\section{WHAT NEXT?}

I have sketched in a few ideas. I propose that interested parties contact me and we will try to take these, and many other, issues further. In the first instance I am prepared to act as a clearing house for an email discussion about the HIA. My email addresses are: SPAN 19739::PNW; BITNET PNW@UK.AC.MAN.JB.STAR.

\section{ACKNOWLEDGEMENTS}

I thank Jan Noordaam for pointing out that a large array is being discussed by Braun and others in the Netherlands and for his impudent suggestions to the conference organiser which led to this talk being given. I thank Rod Davies and Andrew Lyne for discussions about the HIA's scientific goals. So as to come to the subject fresh I did not read the GMRT proposal while writing this paper. But the similarity of many of the issues to those addressed by the GMRT designers, notably Govind Swarup, shows that we will learn a lot from their pioneering efforts over the course of the next ten years. 
G. Swarup: (comment) The major question is to identify some outstanding science objectives - or the "science - drivers" as suggested by you. The antenna design aspects would depend on the above, whether it should be a one square kilometer array or a 100 kilometer long array. Antenna cost can be kept low by identifying sites with low wind etc.

Peter Wilkinson: I couldn't agree more - we must first address the science and the rest will follow! 